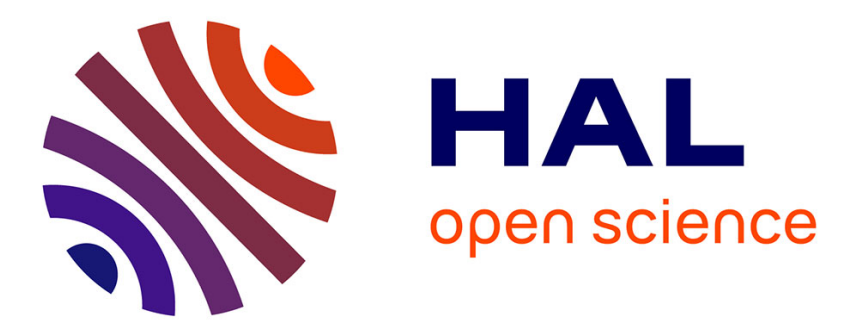

\title{
Stochastic reduced order models for uncertain geometrically nonlinear dynamical systems
}

\author{
M. P. Mignolet, Christian Soize
}

\section{To cite this version:}

M. P. Mignolet, Christian Soize. Stochastic reduced order models for uncertain geometrically nonlinear dynamical systems. Computer Methods in Applied Mechanics and Engineering, 2008, 197 (45-48), pp.3951-3963. 10.1016/j.cma.2008.03.032 . hal-00686140

\section{HAL Id: hal-00686140 \\ https://hal.science/hal-00686140}

Submitted on 7 Apr 2012

HAL is a multi-disciplinary open access archive for the deposit and dissemination of scientific research documents, whether they are published or not. The documents may come from teaching and research institutions in France or abroad, or from public or private research centers.
L'archive ouverte pluridisciplinaire HAL, est destinée au dépôt et à la diffusion de documents scientifiques de niveau recherche, publiés ou non, émanant des établissements d'enseignement et de recherche français ou étrangers, des laboratoires publics ou privés. 


\title{
Stochastic Reduced Order Models For Uncertain Geometrically Nonlinear Dynamical Systems
}

\author{
Marc P. Mignolet ${ }^{1, *}$ and Christian Soize ${ }^{2}$ \\ ${ }^{1}$ Department of Mechanical and Aerospace Engineering \\ Arizona State University, Tempe, AZ 85287-6106, USA - Email: marc.mignolet@asu.edu \\ ${ }^{2}$ Universite Paris-Est, Laboratoire Modelisation et Simulation Multi Echelle, MSME \\ FRE3160 CNRS, 5 bd Descartes, 77454 Marne-la-Vallee, France \\ Email : christian.soize@univ-paris-est.fr
}

\begin{abstract}
A general methodology is presented for the consideration of both parameter and model uncertainty in the determination of the response of geometrically nonlinear structural dynamic systems. The approach is rooted in the availability of reduced order models of these nonlinear systems with a deterministic basis extracted from a reference model (the mean model). Uncertainty, both from parameters and model, is introduced by randomizing the coefficients of the reduced order model in a manner that guarantees the physical appropriateness of every realization of the reduced order model, i.e. while maintaining the fundamental properties of symmetry and positive definiteness of every such reduced order model. This randomization is achieved not by postulating a specific joint statistical distribution of the reduced order model coefficients but rather by deriving this distribution through the principle of maximization of the entropy constrained to satisfy the necessary symmetry and positive definiteness properties. Several desirable features of this approach are that the uncertainty can be characterized by a single measure of dispersion, affects all coefficients of the reduced order model, and is computationally easily achieved. The reduced order modeling strategy and this stochastic modeling of its coefficients are presented in details and several applications to a beam undergoing large displacement are presented. These applications demonstrate the appropriateness and computational efficiency of the method to the broad class of uncertain geometrically nonlinear dynamic systems.
\end{abstract}

\section{KEYWORDS}

Structural uncertainty, uncertainty quantification, random systems, random vibrations, geometric nonlinearity, reduced order models.

\section{INTRODUCTION}

The need to include system uncertainty in dynamic analyses has long been recognized in the context of some specific problems. For example, the response of turbomachinery/engine

\footnotetext{
${ }^{*}$ Corresponding author, Tel. (480) 965-1484, Fax (480) 965-1384.
} 
bladed disks has been known since the late 1960's (e.g. [1]) to be highly sensitive to small bladeto-blade variations in their material/geometrical properties. This lack of robustness has thus motivated numerous stochastic analyses in which the uncertainty/variations in blade properties was introduced through the representation of certain blade characteristics as random variables. This stochastic modeling has however typically been ad-hoc, i.e. only some of the blade properties were considered as random, most notably natural frequencies, based on demonstrated/perceived sensitivity.

With predictive capabilities becoming always faster and allowing always more complex models, the limitations associated with the uncertainty in the parameters of the systems (parameter uncertainty, e.g. in the material properties) and in the computational modeling of the physical system (model uncertainty, e.g. in the finite element representation of fasteners, lap joints, etc., the approximation of the physical geometry, and the introduction of reduced kinematics during the construction of the mean model) now appear clearly in many areas of structural dynamics. Accordingly, it has become quite important to dispose of general methodologies for the inclusion of uncertainty in dynamic analyses, as opposed to the ad-hoc approaches used in the past, and a series of recent investigations have focused on devising such general techniques that are consistent with state-of-the-art computational tools. An attractive approach of this type for parameter uncertainty is the stochastic finite element method (see in particular [2]) in which the random fields characterizing both the uncertain material properties and the response of the system are described by polynomial chaos expansions. Then, given a complete characterization of the uncertain material properties, a similarly complete representation of the stochastic response is obtained. Note however that this probabilistic approach relies on a given computational model and thus does not allow the consideration of model uncertainty. 
A probabilistic approach that does include both parameter and model uncertainty has recently been devised (Soize, [3-5]) and applied/validated (see [6] for a review) on a variety of dynamic problems involving linear structures with possible additional local nonlinearities. The inclusion of parameter and model uncertainty is accomplished in reduced order models of the structure through an appropriate stochastic representation of the elements of its mass, damping, and stiffness matrices. The variations of these random matrices around a baseline model (referred to as the mean model) is characterized by a single measure of dispersion, as opposed to a large number of parameters from statistical distributions. Accordingly, this probabilistic approach has been referred to as nonparametric and thus exhibits the following advantageous properties:

i) includes both model and parameter uncertainty,

ii) is characterized by only a mean reduced order model and a measure of dispersion, and,

iii) is computationally expedient because it relies on reduced order models for the Monte Carlo simulations typically involved in the stochastic analysis of uncertain systems.

These important properties motivate the extension of the nonparametric approach to dynamic systems with distributed, geometric nonlinearity, which is the focus of the present investigation. This extension will rely in particular on recent developments in the formulation of reduced order models of geometrically nonlinear systems (e.g. [7-10]) and will be accomplished in the general framework of linearly elastic geometrically nonlinear structures which encompasses as special cases beams and plates with the von Karman strain definition.

\section{GEOMETRIC NONLINEAR FORMULATION}

While many of the classical structural dynamic problems involving geometric nonlinearity relate to beam, plates, and shells in which the von Karman strain definition is used, 
it is of interest here to demonstrate the general applicability of the nonparametric stochastic modeling approach. To this end, an arbitrary linearly elastic (i.e. with a linear relation between the Green strain and second Piola-Kirchhoff stress tensors) structure undergoing large deformations will be considered in the sequel.

The position vector of a point of the structure will be denoted by $\underline{X}$ in the reference configuration and as $\underline{x}$ in the deformed one so that the displacement vector is $\underline{u}=\underline{x}-\underline{X}$. The deformation gradient tensor $\underline{\underline{F}}$, which is assumed to be orientation preserving, is then defined by its components $F_{i j}$ as

$$
F_{i j}=\frac{\partial x_{i}}{\partial X_{j}}=\delta_{i j}+\frac{\partial u_{i}}{\partial X_{j}}
$$

where $\delta_{i j}$ denotes the Kronecker symbol. Associated with the displacement field $\underline{u}$ are deformations which are characterized by the Green strain tensor $\underline{\underline{E}}$ of components

$$
E_{i j}=\frac{1}{2}\left(F_{k i} F_{k j}-\delta_{i j}\right)
$$

Note in the above equation and in the ensuing ones that summation is implied on all repeated indices.

The equation of motion of the structure is then given by (e.g. see [11])

$$
\frac{\partial}{\partial X_{k}}\left(F_{i j} S_{j k}\right)+\rho_{0} b_{i}^{0}=\rho_{0} \ddot{u}_{i} \quad \text { for } \underline{X} \in \Omega_{0}
$$

where $\underline{\underline{S}}$ denotes the second Piola-Kirchhoff stress tensor, $\rho_{0}(\underline{X})>0$ for all $\underline{X} \in \Omega_{0}$ is the density in the reference configuration, and $\underline{b}^{0}$ is the vector of body forces, all of which are assumed to depend on the coordinates $X_{i}$ and be expressed in the reference configuration in which the structure occupies the domain $\Omega_{0}$. The boundary, $\partial \Omega_{0}$, of the reference configuration 
domain $\Omega_{0}$, is composed of two parts, $\partial \Omega_{0}^{t}$ on which the tractions $\underline{t}^{0}$ are given and $\partial \Omega_{0}^{u}$ on which the displacements are specified. Accordingly, the boundary conditions are

$$
F_{i j} S_{j k} n_{k}^{0}=t_{i}^{0} \quad \text { for } \underline{X} \in \partial \Omega_{0}^{t}
$$

and

$$
\underline{u}=\underline{0} \quad \text { for } \underline{X} \in \partial \Omega_{0}^{u} .
$$

in which $\underline{n}_{0}$ is the external unit normal to $\partial \Omega_{0}^{t}$. Note further in Eqs (3) and (4) that the vectors $\underline{b}^{0}$ and $\underline{t}^{0}$ correspond to the transport of the body forces and tractions applied on the deformed configuration, i.e. $\underline{b}$ and $\underline{t}$, back to the reference configuration. This operation is accomplished through the relations

$$
\underline{b}^{0}=J \underline{b} \quad \text { and } \quad \underline{t}_{0}=\left(\frac{d a}{d A}\right) \underline{t}
$$

where $J$ is the Jacobian of the transformation $\underline{x}=\underline{x}(\underline{X})$, i.e. $J=\operatorname{det}(\underline{\underline{F}})$. Since the deformation gradient satisfies the orientation-preserving condition, we have $J(\underline{X})>0$ for all $\underline{X} \in \Omega_{0}$.Further, the area ratio $d a / d A$ can be expressed evaluated from [12]

$$
\frac{d a}{d A} \underline{n}=J \underline{\underline{F}}^{-T} \underline{N}
$$

where $\underline{N}$ is the unit normal vector to $\partial \Omega_{0}$ at the boundary point $\underline{X}$ and $\underline{n}$ is its counterpart on the deformed configuration.

To complete the formulation of the elastodynamic problem, it remains to specify the constitutive behavior of the material. In this regard, adopting a linear elastic model between the Green strain and second Piola-Kirchhoff stress tensors yields the linear relation

$$
S_{i j}=C_{i j k l} E_{k l}
$$


where the fourth order elasticity tensor $\underline{\underline{C}}$ satisfies the symmetry conditions

$$
C_{i j k l}=C_{j i k l}=C_{i j l k}
$$

as well as

$$
C_{i j k l}=C_{k l i j}
$$

and the positive definiteness property

$$
A_{i j} C_{i j k l} A_{k l}>0
$$

for any non zero second order tensor $\underline{\underline{A}}$.

\section{REDUCED ORDER MODELING}

The previous section has provided the governing equations for the infinite dimensional problem of determining the stress and displacement fields everywhere in the structure considered. Following the discussion of the introduction, it is next desired to construct finite dimensional reduced order models of Eqs (1)-(8) that can be used for a nonparametric stochastic modeling of uncertainty. Before introducing the basis for the reduction, it is necessary to express the problem in its weak form.

To this end, denote by $\underline{v}=\underline{v}(\underline{X})$ a vector function of $\underline{X}$ that is sufficiently differentiable and such that $\underline{v}=\underline{0}$ on $\partial \Omega_{0}^{u}$. Then, the weak formulation of the geometric nonlinear elastodynamic problem of Eqs (3)-(5) is to find the displacement field $\underline{u}$ such that

$$
\int_{\Omega_{0}} \rho_{0} v_{i} \ddot{u}_{i} d \underline{X}+\int_{\Omega_{0}} \frac{\partial v_{i}}{\partial X_{k}}\left(F_{i j} S_{j k}\right) d \underline{X}=\int_{\Omega_{0}} \rho_{0} v_{i} b_{i}^{0} d \underline{X}+\int_{\partial \Omega_{0}^{t}} v_{i} t_{i}^{0} d s
$$

is satisfied for all $v_{i}$ satisfying the above conditions. 
A reduced order model of the nonlinear geometric problem can then be obtained by assuming the displacement field $\underline{u}$ in the form

$$
u_{i}(\underline{X}, t)=\sum_{n=1}^{M} q_{n}(t) U_{i}^{(n)}(\underline{X})
$$

and applying Eq. (12) with $v_{i}=U_{i}^{(m)}$ for $m=1,2, \ldots, M$ where $M$ is the order of the model, i.e. the number of basis functions $U_{i}^{(m)}$ in Eq. (13). After some algebraic manipulations, this process yields

$$
M_{i j} \ddot{q}_{j}+D_{i j} \dot{q}_{j}+K_{i j}^{(1)} q_{j}+K_{i j l}^{(2)} q_{j} q_{l}+K_{i j l p}^{(3)} q_{j} q_{l} q_{p}=F_{i}
$$

where

$$
\begin{gathered}
M_{m n}=\int_{\Omega_{0}} \rho_{0} U_{i}^{(m)} U_{i}^{(n)} d \underline{X} \\
K_{m n}^{(1)}=\int_{\Omega_{0}} \frac{\partial U_{i}^{(m)}}{\partial X_{k}} C_{i k l p} \frac{\partial U_{l}^{(n)}}{\partial X_{p}} d \underline{X} \\
K_{m n p}^{(2)}=\frac{1}{2}\left[\hat{K}_{m n p}^{(2)}+\hat{K}_{p m n}^{(2)}+\hat{K}_{n p m}^{(2)}\right] \\
\hat{K}_{m n p}^{(2)}=\int_{\Omega_{0}} \frac{\partial U_{i}^{(m)}}{\partial X_{j}} C_{i j k l} \frac{\partial U_{r}^{(n)}}{\partial X_{k}} \frac{\partial U_{r}^{(p)}}{\partial X_{l}} d \underline{X} \\
K_{m s n p}^{(3)}=\frac{1}{2} \int_{\Omega_{0}} \frac{\partial U_{i}^{(m)}}{\partial X_{j}} \frac{\partial U_{i}^{(s)}}{\partial X_{k}} C_{j k l w} \frac{\partial U_{r}^{(n)}}{\partial X_{l}} \frac{\partial U_{r}^{(p)}}{\partial X_{w}} d \underline{X}
\end{gathered}
$$

and, finally,

$$
F_{m}=\int_{\Omega_{0}} \rho_{0} U_{i}^{(m)} b_{i}^{0} d \underline{X}+\int_{\partial \Omega_{0}^{t}} U_{i}^{(m)} t_{i}^{0} d s .
$$


Following standard practice, a damping term $D_{i j} \dot{q}_{j}$ has been added in Eq. (14) to represent various dissipation mechanisms in which $\underline{\underline{D}}$ is assumed to be a symmetric positive-definite matrix.

The matrices and tensors involved in Eq. (14) have a variety of properties that arise both from their definitions and the characteristics of the elasticity tensor $\underline{\underline{C}}$, see Eqs (9)-(11). In particular and as expected, it is readily shown that $\underline{\underline{M}}$ and $\underline{\underline{K}}^{(1)}$ are both symmetric and positive definite. Further, it is seen from Eqs (10) and (18) that $\hat{K}_{m n p}^{(2)}=\hat{K}_{m p n}^{(2)}$ which, coupled with the definition of $K_{m n p}^{(2)}$, Eq. (17), implies that $K_{m n p}^{(2)}$ is invariant under any permutation of its indices. Next, it is found from Eqs (9), (10) and (19) that the fourth order tensor $\underline{\underline{K}}^{(3)}$ exhibits the same symmetry properties, i.e. Eq. (9) and (10), as $\underline{\underline{C}}$ and further that it is also positive definite.

In addition to the above properties, which involve each matrix separately, there is also one notable property that involves $\underline{\underline{K}}^{(1)}, \underline{\underline{\hat{K}}}^{(2)}$, and $\underline{\underline{K}}^{(3)}$ together. To demonstrate it, consider the reshaping operating that transforms the $M \mathrm{x} M \mathrm{x} M$ third order tensor $\underline{\underline{\hat{K}}}^{(2)}$ into a $M \mathrm{x} M{ }^{2}$ rectangular array $\underline{\underline{\widetilde{K}}}^{(2)}$ and the $M \mathrm{x} M \mathrm{x} M \mathrm{x} M$ fourth order tensor $\underline{\underline{K}}^{(3)}$ into a $M^{2} \mathrm{x} M^{2}$ square matrix $\underline{\underline{\widetilde{K}}}^{(3)}$. These operations are achieved as follows:

$$
\widetilde{K}_{m J}^{(2)}=\hat{K}_{m n p}^{(2)} \quad \text { with } \quad J=(n-1) M+p
$$

and

$$
\widetilde{K}_{I J}^{(3)}=K_{m s n p}^{(3)} \quad \text { with } \quad I=(m-1) M+s \quad \text { and } \quad J=(n-1) M+p
$$

Next, denote similarly 


$$
Z_{k l}^{(J)}=\frac{\partial U_{r}^{(n)}}{\partial X_{k}} \frac{\partial U_{r}^{(p)}}{\partial X_{l}}
$$

and introduce the $P \mathrm{x} P$ symmetric matrix $\underline{\underline{K}}_{B}$ as

$$
\underline{\underline{K}}_{B}=\left[\begin{array}{ll}
\underline{\underline{K}}^{(1)} & \underline{\underline{\widetilde{K}}}^{(2)} \\
\underline{\underline{\widetilde{K}}}^{(2) T} & 2 \underline{\underline{\widetilde{K}}}^{(3)}
\end{array}\right]
$$

where $P=M+M^{2}$.

It is next desired to demonstrate that $\underline{\underline{K}}_{B}$ is positive definite. To this end, introduce first the $P$-component vector $\underline{W}$ partitioned as

$$
\underline{W}^{T}=\left\lfloor\underline{q}^{T} \quad \underline{V}^{T}\right\rfloor
$$

where $q$ and $\underline{V}$ have $M$ and $M^{2}$ components, respectively. Next, note that

$$
\begin{aligned}
& \underline{W}^{T} \underline{K}_{B} \underline{W}=K_{m n}^{(1)} q_{m} q_{n}+2 \widetilde{K}_{m J}^{(2)} q_{m} V_{J}+2 \widetilde{K}_{I J}^{(3)} V_{I} V_{J} \\
& =\int_{\Omega_{0}}\left\{\left(q_{m} \frac{\partial U_{i}^{(m)}}{\partial X_{k}}\right) C_{i k l p}\left(q_{n} \frac{\partial U_{l}^{(n)}}{\partial X_{p}}\right)+2\left(q_{m} \frac{\partial U_{i}^{(m)}}{\partial X_{j}}\right) C_{i j k l}\left(V_{J} Z_{k l}^{(J)}\right)+\left(V_{I} Z_{j k}^{(I)}\right) C_{j k l w}\left(V_{J} Z_{l w}^{(J)}\right)\right\} d \underline{X}
\end{aligned}
$$

Renaming the dummy indices in the above equation and using the symmetries of Eqs (9) and (10), it is found that

$$
\underline{W}^{T} \underline{K}_{B} \underline{W}=\int_{\Omega_{0}}\left(q_{m} \frac{\partial U_{i}^{(m)}}{\partial X_{j}}+V_{I} Z_{i j}^{(I)}\right) C_{i j k l}\left(q_{n} \frac{\partial U_{k}^{(n)}}{\partial X_{l}}+V_{J} Z_{k l}^{(J)}\right) d \underline{X}
$$

which is positive for all vectors $q$ and $\underline{V}$ given Eq. (11). It is then concluded that the matrix $\underline{\underline{K}}_{B}$ is indeed positive definite. Note that this property also implies the positive definiteness of $\underline{\underline{K}}^{(1)}$ and $\underline{\underline{K}}^{(3)}$ which was stated earlier. 
The availability of the displacement field in the form of Eq. (13) leads to the knowledge of all quantities of interest in both reference and deformed configurations. For example, combining Eqs (1), (2), (8), and (13) leads to the expression for any component of the second Piola-Kirchhoff stress tensor as

$$
S_{i j}=\bar{S}_{i j}+\sum_{m} \hat{S}_{i j}^{(m)} q_{m}+\sum_{m, n} \widetilde{S}_{i j}^{(m, n)} q_{m} q_{n}
$$

where

$$
\begin{gathered}
\bar{S}_{i j}=0 \\
\hat{S}_{i j}^{(m)}=C_{i j k l} \frac{\partial U_{k}^{(m)}}{\partial X_{l}}
\end{gathered}
$$

and

$$
\widetilde{S}_{i j}^{(m, n)}=\frac{1}{2} C_{i j k l} \frac{\partial U_{r}^{(m)}}{\partial X_{k}} \frac{\partial U_{r}^{(n)}}{\partial X_{l}} .
$$

\section{ESTIMATION OF THE REDUCED ORDER MODEL PARAMETERS}

Equations (15)-(20) provide direct expressions for all of the reduced order model parameters given the basis functions $U_{i}^{(m)}(\underline{X})$ and the geometrical and material properties of the structure, e.g. $\rho_{0}, C_{i j k l}, \Omega_{0}$, etc., and thus, technically, complete the reduced order modeling strategy. In practice, however, it is likely that a finite element model of the structure is available and was relied upon to determine the basis functions $U_{i}^{(m)}(\underline{X})$. Then, the integration over $\Omega_{0}$ should be split into integrals over the various elements forming the mesh and the appropriate interpolation functions should be used to evaluate the basis functions $U_{i}^{(m)}(\underline{X})$ and their derivatives. Although fairly straightforward, this effort appears quite cumbersome and may 
require a more detailed knowledge of the inner workings of the finite element package used than may be available, especially for commercially available codes. Accordingly, it would be very desirable to dispose of an indirect approach to determine the various stiffness and mass terms that is compatible with standard finite element packages. One such technique, referred to as the STEP method (STiffness Evaluation Procedure), was initially conceived in [7] and later modified in $[8,9]$.

The fundamental idea behind the STEP approach is to identify the stiffness parameters $K_{i j}^{(1)}, K_{i j l}^{(2)}$, and $K_{i j l p}^{(3)}$ by successive static finite element computations in which the displacement field is prescribed to $\underline{\bar{u}}(X)$ and the required surface tractions $\underline{t}^{0}$ are estimated. The STEP approach starts with the imposition of displacement fields that are proportional to a single basis function, i.e.,

$$
\bar{u}_{i}^{(1)}(\underline{X})=q_{n}^{(1)} U_{i}^{(n)}(\underline{X}) ; \bar{u}_{i}^{(2)}(\underline{X})=q_{n}^{(2)} U_{i}^{(n)}(\underline{X}) \text { and } \bar{u}_{i}^{(3)}(\underline{X})=q_{n}^{(3)} U_{i}^{(n)}(\underline{X})
$$

for each value of $n$ in turn. In these conditions, $q_{n}^{(1)}, q_{n}^{(2)}$, and $q_{n}^{(3)}$ are three constants scaling factors differing from each other and such that the displacements induced $\left(\bar{u}_{i}^{(p)}\right)$ are large enough to induce significant geometric nonlinear effects but small enough to stay within the convergence limits of the finite element code. Inserting the imposed displacement fields of Eq. (32) in the elastostatic equation associated with Eq. (14) implies that

$$
\begin{aligned}
& K_{\text {in }}^{(1)} q_{n}^{(1)}+K_{\text {inn }}^{(2)}\left(q_{n}^{(1)}\right)^{2}+K_{\text {innn }}^{(3)}\left(q_{n}^{(1)}\right)^{3}=F_{i}^{n(1)} \\
& K_{\text {in }}^{(1)} q_{n}^{(2)}+K_{\text {inn }}^{(2)}\left(q_{n}^{(2)}\right)^{2}+K_{\text {innn }}^{(3)}\left(q_{n}^{(2)}\right)^{3}=F_{i}^{n(2)} \\
& K_{\text {in }}^{(1)} q_{n}^{(3)}+K_{\text {inn }}^{(2)}\left(q_{n}^{(3)}\right)^{2}+K_{\text {innn }}^{(3)}\left(q_{n}^{(3)}\right)^{3}=F_{i}^{n(3)}
\end{aligned}
$$


where the force terms $F_{i}^{n(p)}, p=1,2$, or 3, are computed by Eq. (20) from the traction $\underline{t}^{0}$ predicted in each case by the finite element code with body force $\underline{b}^{0}(\underline{X})=\underline{0}$ for all $\underline{X} \in \Omega_{0}$. Equations (33)-(35) represent for each $i$ and $n$ a set of three linear equations in the unknown $K_{i j}^{(1)}, K_{i n n}^{(2)}$, and $K_{i n n n}^{(3)}$ which is readily solved.

The next stage of the STEP algorithm focuses on the determination of the parameters $K_{\text {imn }}^{(2)}, K_{\text {immn }}^{(3)}$, and $K_{\text {imnn }}^{(3)}$ (and their permutations of indices, see discussion on properties of $\underline{\underline{K}}^{(2)}$ and $\underline{\underline{K}}^{(3)}$ ) with $m \neq n$. This computation is again achieved by imposing displacement fields and determining the necessary tractions $\underline{t}^{0}$ and associated force terms $F_{i}$, Eq. (20). However, the procedure is slightly different from the one above in that involving the parameters $K_{\text {imn }}^{(2)}, K_{\text {immn }}^{(3)}$, and $K_{\text {imnn }}^{(3)}$ requires a displacement field that has components in both $U_{i}^{n}$ and $U_{i}^{m}$, i.e.

$$
\begin{gathered}
\bar{u}_{i}^{(4)}(\underline{X})=q_{n}^{(4)} U_{i}^{(n)}(\underline{X})+q_{m}^{(4)} U_{i}^{(m)}(\underline{X}) \quad ; \quad \bar{u}_{i}^{(5)}(\underline{X})=q_{n}^{(5)} U_{i}^{(n)}(\underline{X})+q_{m}^{(5)} U_{i}^{(m)}(\underline{X}) \\
\text { and } \\
\bar{u}_{i}^{(6)}(\underline{X})=q_{n}^{(6)} U_{i}^{(n)}(\underline{X})+q_{m}^{(6)} U_{i}^{(m)}(\underline{X})
\end{gathered}
$$

Expressing the reduced order model governing equation, Eq. (14), for these 3 displacement fields and the associated force terms $F_{i}^{n(p)}, p=4,5$, and 6 , yields a set of three equations for each $i$, $m$, and $n$ which is readily solved to obtain the parameters $K_{i m n}^{(2)}, K_{i m m n}^{(3)}$, and $K_{i m n n}^{(3)}$. The choice of scaling factors $q_{n}^{(4)}=q_{n}^{(5)}=-q_{n}^{(6)}$ and $q_{m}^{(4)}=-q_{m}^{(5)}=-q_{m}^{(6)}$ does lead to some simplifications of the equations. 
The last stage of the STEP algorithm is concerned with the evaluation of the coefficients $K_{i m n s}^{(3)}$ (and its permutations of indices) for $m, n$, and $s$ all different. This effort is readily achieved from the above results using the final displacement field

$$
\bar{u}_{i}^{(7)}(\underline{X})=q_{n}^{(7)} U_{i}^{(n)}(\underline{X})+q_{m}^{(7)} U_{i}^{(m)}(\underline{X})+q_{s}^{(7)} U_{i}^{(s)}(\underline{X})
$$

and the associated force term $F_{i}^{(7)}$.

The series of finite element computations performed for the evaluation of the stiffness parameters can serve as well for the estimation of the coefficients $\bar{S}_{i j}(\underline{X}), \hat{S}_{i j}^{(m)}(\underline{X})$, and $\widetilde{S}_{i j}^{(m, n)}(\underline{X})$ of the reduced order model for the stress $S_{i j}$ at location $\underline{X}$ provided that the values of this stress are output during each run. The procedure is similar to the one conducted above and will not be repeated here.

It should however be noted that the stress is a second order polynomial of the generalized coordinates $q_{n}$ as compared to the cubic nature of the nonlinear stiffness terms. Thus, many fewer computations would be necessary to estimate $\bar{S}_{i j}(\underline{X}), \hat{S}_{i j}^{(m)}(\underline{X})$, and $\widetilde{S}_{i j}^{(m, n)}(\underline{X})$ than are actually performed. The redundant cases can then be used to assess the accuracy of the estimates of these stress coefficients and thus provide a first mean to quantify the robustness of the STEP algorithm. A second perspective on this robustness can be obtained from a check of the symmetries of the tensors $\underline{\underline{K}}^{(1)}, \underline{\underline{K}}^{(2)}$, and $\underline{\underline{K}}^{(3)}$ which were not used in the determination of their components. For example, the properties $K_{i j}^{(1)}=K_{j i}^{(1)}$ were not relied upon in Eqs (35)(37). Similarly, the symmetry $K_{i j k}^{(2)}=K_{k i j}^{(2)}$ was not used in the computations while the property $K_{i j k}^{(2)}=K_{i k j}^{(2)}$ was assumed. A similar discussion also holds with the elements of $\underline{\underline{K}}^{(3)}$. A 
detailed discussion of these issues in connection with a flat plate (see [8]) has shown that there exists a fairly broad range of the scaling factors $q_{i}^{(p)}$ over which the estimates of the stiffness coefficients are accurate and stable.

Once the above computations have been performed, all coefficients of the reduced order model equations of motion, Eq. (14), are known but it still remains to evaluate the third order tensor $\hat{K}_{m n p}^{(2)}$ that appears in the matrix $\underline{\underline{K}}_{B}$ through its reshaped array $\widetilde{K}^{(2)}$. To this end, note that Eq. (17) implies that

$$
K_{i j l}^{(2)} q_{j} q_{l}=\frac{1}{2} \frac{\partial}{\partial q_{i}}\left[\hat{K}_{j l p}^{(2)} q_{j} q_{l} q_{p}\right]
$$

and thus the coefficients $\hat{K}_{m n p}^{(2)}$ can be evaluated through the integration of/finding the potential associated with $K_{i j l}^{(2)} q_{j} q_{l}$.

\section{NONPARAMETRIC STOCHASTIC MODELING OF UNCERTAINTY}

As discussed in the introduction, there are two particular types of uncertainty to be considered in structural dynamic models: parameter uncertainty and model uncertainty. The former is associated with variations of the material properties of the structure that arise from the manufacturing process, in service operation, and the modeling process. At the contrary, model uncertainty recognizes that the computational model is a simplified representation of the physical structure. For example, components such as fasteners (rivets, bolts,...) and joints (lap joints, welds, ...) etc. are usually only approximately represented, i.e. modeled. A similar discussion holds with the geometry of the structure which differs slightly from the computational one, plates may be slightly warped, beams out of straight, etc. Thus, deviations in the behaviors of actual 
structures and their computational counterparts are expected and variable and form the model uncertainty.

The consideration of parameter uncertainty in the infinite dimensional problem of Eqs (1)-(8) is in principle quite straightforward, it can be achieved by letting the material properties $\rho_{0}, \underline{\underline{C}}, \ldots$ be random field. The inclusion of model uncertainty in the same framework is however very challenging. For example, the consideration of variations of geometry would require changing the computational model (i.e. the finite element mesh) for every realization of the geometry. The consideration of other model issues, as related to the approximate representation of joints, fasteners, etc. appears even more difficult if at all possible.

On the contrary, the consideration of uncertainty, from parameters or model, appears much more straightforward in the reduced order model, as it is characterized by a finite number of mass and stiffness coefficients which can be treated as random variables and are physically expected to be correlated. In addition to those coefficients, the reduced order model also involves the basis functions $U_{i}^{(n)}$ and it is worthwhile to ask whether that basis should be deterministic (i.e. related to the mean model) or random (e.g. based on the full computational model with some parameter uncertainty). The most significant advantage of using a random basis would be to obtain certain special properties of the uncertain reduced order model, e.g. diagonal nature of some of the matrices involved. However, the inclusion of model uncertainty would likely destroy these special features. On the contrary, the use of a deterministic basis is computationally efficient as it needs to be determined only once and focuses the uncertainty of the reduced order model on its coefficients. On the basis of this discussion, deterministic basis functions will be adopted in the sequel but their choice must be such that the response of the uncertain systems, not just the mean one, is well represented. Additional comments in this respect will be made in the Numerical Results section. 
Parameter and model uncertainty will thus be included through randomizing the various mass, damping and stiffness coefficients of Eq. (14), see Eqs (15)-(20). This apparently simple statement has far ranging implications as a complete characterization of this ensemble of random variables requires the specification of their joint probability density function, an information which is unlikely to be available in any practical application. A first approach to resolve this difficulty is to allow only some of the coefficients to be uncertain as was done in the ad-hoc strategies discussed in the Introduction. Clearly such an approach does not have the accuracy and generality required here. A second approach might be to specify the form of this distribution with unknown parameters to be estimated. Even with a single parameter per uncertain coefficient to describe its variations, there would be a very large number of such parameters to estimate especially given the generally poor knowledge on the uncertainty in physical systems.

A third approach, which is the one adopted here, is to rely on a higher principle to derive the necessary joint probability density function. As discussed by Soize [3-6], the maximum entropy principle provides such a framework and leads to statistical distributions that place particular emphasis on "larger" deviations from the mean value, a desirable feature to assess the robustness of a design to uncertainty. The maximization of the entropy must however be achieved carefully to guarantee the physical meaningfulness of the ensemble of mass, damping, and stiffness coefficients simulated, especially in view of the emphasis on the tail of the distribution just stated. Physical meaningfulness of the mass, damping, and stiffness coefficients of the reduced order model of Eq. (14) implies here that these coefficients satisfy all properties that are expected from Eq. (14) for an arbitrary dynamic system, i.e.

(1) the stated symmetry properties of the tensors $\underline{\underline{M}}, \underline{\underline{D}}, \underline{\underline{\breve{K}}}^{(1)}, \underline{\underline{K}}^{(2)}$, and $\underline{\underline{\breve{K}}}^{(3)}$

(2) the positive definiteness of the matrices $\underline{\underline{M}}$, $\underline{\underline{D}}$, and $\underline{\underline{K}}_{B}$ 
(3) the nonsingularity of the matrices $\underline{\underline{M}}, \underline{\underline{D}}$, and $\underline{\underline{K}}_{B}$ (if true for the mean model).

Note in the above conditions that $\underline{\underline{M}}$ is the uncertain mass matrix resulting from the consideration of uncertainty in contrast to the deterministic mass matrix $\underline{\underline{M}}$ of the mean model. A similar convention was also used above for $\underline{\underline{D}}, \underline{\underline{K}}_{B}, \underline{\underline{\breve{K}}}^{(1)}, \underline{\underline{K}}^{(2)}$, and $\underline{\underline{K}}^{(3)}$ and will be employed in the sequel to distinguish all random quantities.

The consideration of uncertainty in the reduced order model of Eq. (14) is then achieved by analyzing the response of the uncertain system

$$
\breve{M}_{i j} \breve{q}_{j}+\breve{D}_{i j} \grave{\dot{q}}_{j}+\breve{K}_{i j}^{(1)} \breve{q}_{j}+\breve{K}_{i j l}^{(2)} \breve{q}_{j} \breve{q}_{l}+\breve{K}_{i j l p}^{(3)} \breve{q}_{j} \breve{q}_{l} \breve{q}_{p}=\breve{F}_{i}
$$

Note as well that the excitation $F_{i}$ of Eq. (14) has been replaced by the uncertain term $\breve{F}_{i}$ in Eq. (39). This randomization of the excitation reflects the possible uncertain nature of the transport of the specified traction from the (uncertain) deformed configuration back to the (deterministic) reference one.

In his original formulation of the nonparametric stochastic modeling approach, Soize $[3,4]$ addressed the problem of determining the joint probability density function $p_{\underline{\underline{A}}}(\underline{\underline{a}})$ of the elements $\breve{A}_{i j}$ of a random symmetric positive definite $\bar{n} \times \bar{n}$ matrix $\underline{\underline{A}}$ that maximizes the entropy

$$
S=-\int_{\Omega} p_{\underline{\underline{A}}}(\underline{\underline{a}}) \ln p_{\underline{\underline{A}}}(\underline{\underline{a}}) d \underline{\underline{a}}
$$

in which $d \underline{\underline{a}}$ is the appropriate volume element. Further, $\Omega$ denotes the domain of support of $p_{\underline{\underline{A}}}(\underline{\underline{a}})$ which should be such that any matrix $\underline{\underline{a}} \in \Omega$ is symmetric and positive definite. These conditions are equivalent to stating that $\underline{\underline{a}}$ admits a Cholesky decomposition $\underline{\underline{a}}=\underline{\underline{L}} \underline{\underline{L}}^{T}$ so that 


$$
\Omega=\left\{\underline{\underline{a}}=\underline{\underline{L}} \underline{\underline{L}}^{T} ; L_{i j}, i, j=1, \ldots, \bar{n}:\left[L_{i j} \in(-\infty,+\infty), i>j\right] \cap\left[L_{i i} \in[0,+\infty)\right]\right\} .
$$

The maximization of $S$, Eq. (40), must be achieved under the following constraints

$$
\begin{gathered}
\int_{\Omega} p_{\underline{\breve{A}}}(\underline{\underline{a}}) d \underline{\underline{a}}=1 \\
E[\underline{\underline{A}}]=\int_{\Omega} \underline{\underline{a}} p_{\underline{\underline{A}}}(\underline{\underline{a}}) d \underline{\underline{a}}=\underline{\underline{A}}
\end{gathered}
$$

and

$$
\int_{\Omega} \ln [\operatorname{det}(\underline{\underline{a}})] p_{\underline{\underline{A}}}(\underline{\underline{a}}) d \underline{\underline{a}}=v(\text { finite })
$$

where $E[$.$] denotes the operation of mathematical expectation and \operatorname{det}(\underline{\underline{W}})$ is the determinant of an arbitrary matrix $\underline{\underline{W}}$. The first two of the above constraints correspond to the normalization of the total probability to 1 (Eq. (42)) and the specification of the mean matrix (Eq. (43)). The third one, Eq. (44), implies the existence of the mean squared Frobenius norm of the inverse matrix $\underline{\breve{A}}^{-1}$ (see $[3,4]$ for discussion) and thus guarantees the nonsingularity of $\underline{\underline{A}}$ for mean square norm, i.e. $E\left[\left\|\breve{A}^{-1}\right\|_{F}^{2}\right]<+\infty$, where \|\|$_{F}$ denotes the Frobenius norm of matrices.

The determination of the probability density function maximizing Eq. (40) while satisfying the constraints of Eqs (42)-(44) was accomplished in [3,4] by calculus of variations with Lagrange multipliers. The resulting stochastic description of $\underline{\underline{A}}$ is most easily stated in terms of the random lower triangular matrix $\underline{\underline{H}}$ such that

$$
\underline{\underline{\breve{A}}}=\underline{\underline{\underline{L}}} \underline{\underline{\underline{H}}} \underline{\underline{H}}^{T} \underline{\underline{L}}^{T}
$$


where $\underline{\underline{L}}$ is any decomposition, e.g. Cholesky, of $\underline{\underline{A}}$ satisfying $\underline{\underline{A}}=\underline{\underline{\underline{L}}} \underline{\underline{L}}^{T}$. Specifically, it was found $[3,4]$ that

$$
p_{\underline{\underline{\underline{H}}}}(\underline{\underline{h}})=\prod_{i=1}^{\bar{n}}\left\{\bar{C}_{i} h_{i i}^{p(i)} \exp \left[-\mu_{i i} h_{i i}^{2}\right]\right\} \times \prod_{i=1}^{\bar{n}} \prod_{l=1}^{i-1}\left\{\bar{C}_{i l} \exp \left[-\mu_{i i} h_{i l}^{2}\right]\right\}
$$

where $\bar{C}_{i}, i=1, \ldots, \bar{n}$, and $\bar{C}_{i l}, i=1, \ldots, \bar{n} ; l=1, \ldots, i-1$, are appropriate normalization constants and

$$
\begin{gathered}
p(i)=\bar{n}-i+2 \lambda-1 \\
\mu_{i i}=\frac{\bar{n}+2 \lambda-1}{2} .
\end{gathered}
$$

It is concluded from Eq. (46) that:

(i) the elements $\breve{H}_{i l}, i>l$, are all independent of each other and independent of the elements $\breve{H}_{i i}$. Further, they are normally distributed with mean 0 and standard deviation $\sigma_{i l}=1 / \sqrt{2 \mu_{i i}}$.

(ii) the elements $\breve{H}_{i i}$ are all independent of each other. Further, they are distributed according to

$$
p_{\breve{H}_{i i}}\left(h_{i i}\right)=\bar{C}_{i} h_{i i}^{p(i)} \exp \left[-\mu_{i i} h_{i i}^{2}\right], h_{i i} \geq 0
$$

where

$$
\bar{C}_{i}=\frac{2 \mu_{i i}^{[p(i)+1] / 2}}{\Gamma((p(i)+1) / 2)}
$$

and $\Gamma($.$) denotes the Gamma function.$

The generation of samples of $\breve{H}_{i i}$ is simplified by considering the variable $\breve{Y}_{i i}=\mu_{i i} \breve{H}_{i i}^{2}$. Proceeding with the change of variables, it is found that the probability density function of $\breve{Y}_{i i}$ is

$$
p_{\breve{Y}_{i i}}\left(y_{i i}\right)=\frac{y_{i i}^{(p(i)-1) / 2}}{\Gamma[(p(i)+1) / 2]} \exp \left[-y_{i i}\right], \quad y_{i i} \geq 0 .
$$

Accordingly, it is found that $\breve{Y}_{i i}$ is a Gamma distributed random variable for which efficient 
simulation algorithms exist, e.g. see [13]. Once a sample of $\breve{Y}_{i i}$ has been simulated according to the Gamma distribution, the corresponding value of $\breve{H}_{i i}$ is found as

$$
\breve{H}_{i i}=\sqrt{\frac{\breve{Y}_{i i}}{\mu_{i i}}}
$$

where $\mu_{i i}$ is given by Eq. (48).

The parameter $\lambda$ which appears in Eq. (47) and (48) is in fact the Lagrange multiplier associated with the constraint of Eq. (44) and could be evaluated from this condition. However, since Eq. (44) was enforced to ensure an appropriately flat zero of $p_{\underline{\underline{A}}}(\underline{\underline{a}})$ near its singular boundary (i.e. an appropriately flat zero of $p_{\breve{H}_{i i}}\left(h_{i i}\right)$ at $h_{i i}=0$ ), it is more appropriate to use the parameter $\lambda$ to control the variations of the random matrices $\underline{\underline{A}}$ from their mean value $\underline{\underline{A}}$, as will be clarified in the Numerical Results section. In particular, the parameter $\lambda$ can also be expressed [4] as

$$
\lambda=\frac{\left(1-\delta^{2}\right)}{2 \delta^{2}} \bar{n}+\frac{\left(1+\delta^{2}\right)}{2 \delta^{2}}
$$

in which $\delta$ is the coefficient of variation of the random matrix $\underline{\underline{\breve{G}}}=\underline{\underline{\breve{H}}} \underline{\underline{\breve{H}}}^{T}$, i.e. $\delta^{2}=\frac{1}{\bar{n}} E\left[\|\underline{\underline{G}}-\underline{\underline{I}}\|_{F}^{2}\right]$ where $\underline{\underline{I}}$ is the identity matrix.

Equations (45)-(52) form here the core methodology for the simulation of the uncertain mass, damping, and stiffness coefficients of Eq. (39) as described below. It should be noted that Eq. (45)-(52) follow the original formulation of the nonparametric approach of Ref. [3] but that extensions of the methodology have been carried out which permit a more detailed modeling of the structural uncertainty present. For example, knowledge of the level of uncertainty of multiple natural frequencies (and related data) can also be included, see [14] for details. A more complex 
structure of the uncertain mass, stiffness, and damping matrices generated, e.g. with the capability of exhibiting topological zeros, can also be simulated (see [15]). The use of these extended uncertainty models would closely follow the steps described here except for Eq. (45)(52) which would be replaced by different relations, see $[14,15]$ for details.

For the uncertain mass matrix $\underline{\underline{M}}$ :

A Cholesky decomposition of the mass matrix of the mean model $\underline{\underline{M}}$ is first performed as

$$
\underline{\underline{M}}=\underline{\underline{L}}_{M} \underline{\underline{L}}_{M}^{T}
$$

Next, a value $\lambda=\lambda_{M}$ is specified and an ensemble of $\bar{n} \times \bar{n}$ lower triangular matrices $\underline{\underline{\breve{H}}}_{M}$, with $\bar{n}=M$, are generated according to Eq. (45)-(52). For each realization of $\underline{\underline{H}}_{M}$, the corresponding sample of the mass matrix $\underline{\underline{M}}$ is generated as

$$
\underline{\underline{\underline{M}}}=\underline{\underline{L}}_{M} \underline{\underline{\breve{H}}}_{M} \underline{\underline{\breve{H}}}_{M}^{T} \underline{\underline{L}}_{M}^{T}
$$

For the uncertain damping matrix $\underline{\underline{D}}$ :

A Cholesky decomposition of the damping matrix of the mean model $\underline{\underline{D}}$ is first performed as

$$
\underline{\underline{D}}=\underline{\underline{L}}_{D} \underline{\underline{L}}_{D}^{T}
$$

Next, a value $\lambda=\lambda_{D}$ is specified and an ensemble of $\bar{n} \times \bar{n}$ lower triangular matrices $\underline{\underline{H}}_{D}$, with $\bar{n}=M$, are generated according to Eq. (45)-(52). For each realization of $\underline{\underline{H}}_{D}$, the corresponding sample of the damping matrix $\underline{\underline{D}}$ is generated as

$$
\underline{\underline{D}}=\underline{\underline{L}}_{D} \stackrel{\breve{H}}{D} \underline{\underline{\breve{H}}}^{T} \underline{\underline{L}}^{T}
$$


For the uncertain stiffness tensors $\underline{\underline{K}}^{(1)}, \underline{\underline{K}}^{(2)}$, and $\underline{\underline{K}}^{(3)}$ :

A Cholesky decomposition of the matrix $\underline{\underline{K}}_{B}$ of the mean model is first performed as

$$
\underline{\underline{K}}_{B}=\underline{\underline{L}}_{K} \underline{\underline{L}}_{K}^{T}
$$

Next, a value $\lambda=\lambda_{K}$ is specified and an ensemble of $\bar{n} \times \bar{n}$ lower triangular matrices $\underline{\underline{H}}_{K}$, with $\bar{n}=M+M^{2}$, are generated according to Eq. (45)-(52). For each realization of $\underline{\underline{H}}_{K}$, the corresponding sample of the matrix $\underline{\underline{K}}_{B}$ is generated as

$$
\underline{\underline{K}}_{B}=\overline{\underline{L}}_{K} \underline{\underline{H}}_{K} \underline{\underline{H}}_{K}^{T} \underline{\underline{L}}_{K}^{T}
$$

The partitioning of the uncertain matrix $\underline{\underline{K}}_{B}$ is consistent with the one of its mean value, i.e. Eq. (24), and thus permits to extract the corresponding uncertain matrices $\underline{\underline{K}}^{(1)}, \underline{\underline{\widetilde{K}}}^{(2)}$, and $\underline{\underline{\widetilde{K}}}^{(3)}$. No further manipulation is needed in connection with the linear stiffness matrix $\underline{\underline{K}}^{(1)}$ but $\underline{\underline{\widetilde{K}}}^{(2)}$ and $\underline{\underline{\widetilde{K}}}^{(3)}$ must next be reshaped into uncertain third and fourth order tensors $\underline{\underline{\hat{K}}}^{(2 N S)}$ and $\underline{\underline{K}}^{(3 N S)}$ as in Eq. (21) and (22) for $\underline{\underline{\hat{K}}}^{(2)}$ and $\underline{\underline{K}}^{(3)}$ from $\underline{\underline{\widetilde{K}}}^{(2)}$ and $\underline{\underline{\widetilde{K}}}^{(3)}$. Note that the additional superscript $N S$ indicates that these matrices do not exhibit the appropriate symmetries, for example $\widehat{\hat{K}}_{m n p}^{(2 N S)}$ is in general not equal to $\overline{\hat{K}}_{m p n}^{(2 N S)}$. This issue is easily resolved and tensors $\underline{\underline{\hat{K}}}^{(2)}$ and $\underline{\underline{K}}^{(3)}$ with the appropriate symmetries are then generated as

$$
\breve{\hat{K}}_{m n p}^{(2)}=\frac{1}{2}\left(\overline{\hat{K}}_{m n p}^{(2 N S)}+\overline{\hat{K}}_{m p n}^{(2 N S)}\right)
$$

and 


$$
\breve{K}_{m s n p}^{(3)}=\frac{1}{4}\left(\breve{K}_{m s n p}^{(3 N S)}+\breve{K}_{s m n p}^{(3 N S)}+\breve{K}_{m s p n}^{(3 N S)}+\breve{K}_{s m p n}^{(3 N S)}\right)
$$

since the property $\breve{K}_{m s n p}^{(3 N S)}=\breve{K}_{n p m s}^{(3 N S)}$ already exists as a result of the symmetry of $\underline{\underline{K}}_{B}$, see Eq. (59) and (24).

It remains finally to obtain the realization of the third order tensor $\underline{\underline{K}}^{(2)}$ from $\underline{\underline{\hat{K}}}^{(2)}$. This is achieved as in Eq. (17), i.e.

$$
\breve{K}_{m n p}^{(2)}=\frac{1}{2}\left(\breve{\hat{K}}_{m n p}^{(2)}+\breve{\hat{K}}_{p m n}^{(2)}+\breve{\hat{K}}_{n p m}^{(2)}\right)
$$

\section{NUMERICAL RESULTS}

To demonstrate the above concepts, a beam of dimensions $0.2286 \mathrm{~m}$ long, $0.0127 \mathrm{~m}$ wide, and $7.75 \times 10^{-4} \mathrm{~m}$ high was discretized by the finite element method (with MSC NASTRAN) into 40 CBEAM elements of equal lengths (selected computations were repeated with a 160 element model which confirmed the adequacy of the 40 element model). The two ends of the beam were assumed to be fully clamped. This model thus included 234 degrees-of-freedom. The beam material was high-carbon steel with a Young's modulus of 205,000 MPa, a shear modulus of $80,000 \mathrm{MPa}$, and a mass density of $7,875 \mathrm{~kg} / \mathrm{m}^{3}$. The damping in the mean system was assumed to be classical with a damping ratio of $2 \%$ on all modes.

The beam was assumed to be excited by a single, deterministic, concentrated force acting on its middle in the direction perpendicular to the beam axis in the undeformed configuration, see Fig. 1 for a representative time history of the force and Fig. 2 for its frequency content, i.e. flat in the range $[-2000,+2000] \mathrm{Hz}$.

The time marching of the response of the reduced order models (mean and uncertain) was achieved with an unconditionally stable Newmark- $\beta$ algorithm (e.g. see [16]) in which the nonlinear algebraic equations were solved by a fixed point algorithm. The time step for the most 
of the computations was set at $\Delta t=5 \times 10^{-5} \mathrm{~s}$ and the computations were carried out for $n_{\text {tot }}=15000$ time steps. No convergence problem with the fixed point algorithm was encountered with the above time step.

The basis for the reduced order modeling effort included the first $n_{t}$ transverse linear modes and $n_{i}$ in-plane linear modes exhibiting the natural frequencies shown in Table 1 . The selection of the appropriate values of $n_{t}$ and $n_{i}$ was performed in each case by monitoring the convergence of the total modal signal energy of the mean model:

$$
E\left(n_{t}, n_{i}\right)=\sum_{n=1}^{n_{t o t}} \sum_{i=1}^{M} q_{i}^{2}(n \Delta t)
$$

where $M=n_{t}+n_{i}$ is the order of the reduced order model. In general, it was found that the selection $n_{t}=10$ and $n_{i}=12$ led to convergence. For the cases in which these parameters were changed, their values will be stated explicitly. It should be noted that the mean system is symmetric with respect to the location of the force and thus the second, fourth, and sixth transverse modes, which are antisymmetric, will not appear in the mean model response. However, the introduction of uncertainty in the beam reduced order model will in general break the symmetry and induce contributions of the response on these antisymmetric transverse modes. The same observation holds in regards to the in-plane modes.

The direction of the force induced transverse motions in the weak bending direction (" $z$ ") with much smaller motions along the beam axis (" $x$ ") taking place by nonlinear interaction. The magnitude of these displacements satisfied the assumptions of the von Karman strain definition, i.e. 


$$
\begin{gathered}
\varepsilon_{x}=\frac{\partial u_{1}}{\partial x}+\frac{1}{2}\left(\frac{\partial u_{3}}{\partial x}\right)^{2} \quad \varepsilon_{y}=\frac{\partial u_{2}}{\partial y}+\frac{1}{2}\left(\frac{\partial u_{3}}{\partial y}\right)^{2} \\
\varepsilon_{z}=\frac{\partial w}{\partial z}=0 \\
\gamma_{x y}=\frac{1}{2}\left[\frac{\partial u_{1}}{\partial y}+\frac{\partial u_{2}}{\partial x}+\left(\frac{\partial u_{3}}{\partial x}\right)\left(\frac{\partial u_{3}}{\partial y}\right)\right] \quad \gamma_{x z}=\frac{1}{2}\left[\frac{\partial u_{1}}{\partial z}+\frac{\partial u_{3}}{\partial x}\right] \\
\gamma_{y z}=\frac{1}{2}\left[\frac{\partial u_{2}}{\partial z}+\frac{\partial u_{3}}{\partial y}\right]
\end{gathered}
$$

Note that the above expressions result from Eq. (2) by ignoring the second order terms in $u_{1}$ and $u_{2}$. In fact, the displacement $u_{2}$ is identically zero for this problem. The close applicability of the von Karman strain definition led to a peculiarity of the matrix $\underline{\underline{K}}_{B}$. Specifically, the absence of second order terms in $u_{1}$ in the displacement-strain relation can be shown to imply the vanishing of all terms $K_{m n p}^{(2)}$ and $K_{m s n p}^{(3)}$ in which two or more indices $m, n, p$, and $s$ refer to inplane modes. This property leads theoretically to a matrix $\underline{\underline{K}}_{B}$ exhibiting a significant number of zero eigenvalues and computationally (given the finite accuracy of the STEP algorithm) to the same number of small generally both positive and negative eigenvalues. Clearly, the negative eigenvalues are unphysical (see Eq. (27)) and must be removed before the consideration of uncertainty takes place. In doing so, it is necessary to demonstrate that these computationally negative values do not have an effect on the response. This check was accomplished on the mean model by computing the response with the identified $\underline{\underline{K}}_{B}$ matrix (exhibiting small negative eigenvalues) and the singular value decomposition of $\underline{\underline{K}}_{B}$ in which only the positive eigenvalues were retained. In all cases considered, the match of the two transverse responses at the middle point were in a visually perfect agreement over both entire time history and frequency range.

The elimination of the negative eigenvalues of $\underline{\underline{K}}_{B}$ by spectral decomposition led to the modified matrix ${\underline{\underline{K^{\prime}}}}_{B}$ defined as 


$$
{\underline{\underline{K^{\prime}}}}_{B}=\underline{\underline{\Phi}} \underline{\underline{\Lambda}} \underline{\underline{\Phi}}^{T}
$$

where $\underline{\underline{\Phi}}$ denotes the matrix whose $n_{r}<M+M^{2}$ columns are the eigenvectors of $\underline{\underline{K}}_{B}$ corresponding to its positive eigenvectors. Further, $\underline{\underline{\Lambda}}$ is the diagonal matrix containing the $n_{r}$ positive eigenvalues of $\underline{\underline{K}}_{B}$. Then, following Soize [5], the simulation of uncertain matrices $\underline{\underline{K}}_{B}$ is achieved by first generating an ensemble of $\bar{n} \times \bar{n}$ lower triangular matrices $\underline{\underline{H}} K r$, with $\bar{n}=n_{r}$ and $\lambda=\lambda_{K r}$ according to Eq. (45)-(52). Finally, the corresponding uncertain matrices $\underline{\underline{K}}_{B}$ are obtained as

$$
\underline{\underline{K}}_{B}=\left(\underline{\underline{\Phi}} \underline{\underline{\Lambda}}^{1 / 2}\right) \underline{\underline{H}}_{K r} \underline{\underline{\breve{H}}}_{K r}^{T}\left(\underline{\underline{\Phi}} \underline{\underline{\Lambda}}^{1 / 2}\right)^{T}
$$

To enable a physical comparison between different cases, the various parameters $\lambda$ should be selected to achieve the same physical measure of variation of the reduced order model. In the present investigation, the measure of variation specified related to the first natural frequency. More specifically, a 4\% mean square variation of the first natural frequency of the uncertain system $\left(\Omega_{1}\right)$ around its corresponding value for the mean model $\left(\omega_{1}\right)$ was enforced. That is,

$$
E\left[\left(\Omega_{1}-\omega_{1}\right)^{2}\right]=\left(0.04 \omega_{1}\right)^{2}
$$

The evaluation of the parameter $\lambda$ from this condition was achieved in a trial and error strategy: for a value of $\lambda$, an ensemble of reduced order models were generated and the corresponding population of the first natural frequency of the uncertain linear system were determined. An estimate of $E\left[\left(\Omega_{1}-\omega_{1}\right)^{2}\right]$ was then obtained and the process was repeated until Eq. (67) was satisfied.

Once the appropriate value of $\lambda$ has been determined, the generation of samples of the 
mass, damping, and stiffness coefficients of the uncertain reduced order model can be achieved and the corresponding realizations of the response time histories $\underline{q}$ can be obtained by numerical integration of Eq. (39). But how many such samples should be generated? This issue was resolved here my monitoring, for each case separately, the convergence of the estimate of the mean modal signal energy

$$
\frac{1}{n_{\text {sam }}} \sum_{i=1}^{n_{\text {sam }}} \sum_{n=1}^{n_{\text {tot }}} \underline{\breve{q}}^{(i) T}(n \Delta t) \underline{\underline{q}}^{(i)}(n \Delta t) \rightarrow E\left[\underline{\breve{q}}^{T} \underline{\breve{q}}\right]
$$

where $\underline{\breve{q}}^{(i)}(n \Delta t)$ denotes the vector of the $n_{t}+n_{i}$ generalized coordinates of sample $i$ at the $n$th time step.

A first goal of the present numerical efforts was to assess the effects of geometric nonlinearity on the response of the uncertain beams. To this end, three different cases were considered with uncertainty on the stiffness terms. First, a linear computation was carried out by ignoring the quadratic and cubic terms in Eq. (39) and determining the response to the excitation of Figs 1 and 2. Second, a fully nonlinear computation was carried out with the same excitation. Finally, this last set of computations was repeated at a higher excitation level.

Shown in Figs 3 and 4 is the displacement of the mid point of the beam predicted from the linear mean model both in time (Fig. 3) and in frequency (Fig. 4) with 12 in-plane modes and 10 transverse. The sharp drop in energy right at the cut-off frequency $(2000 \mathrm{~Hz})$ and the sharp resonance peaks are distinctive of the linear system. A convergence study of the mean modal signal energy of the uncertain linear system, see Fig. 5, demonstrated that 600 samples were sufficient for convergence. Then, various statistics of the response could be obtained. Of particular interest here was the physical deflection of the center of the beam, more specifically its frequency content. Shown in Fig. 6 are the mean, 5th, and 95th percentiles of the spectrum of the uncertain response of the middle of the beam. These curves were obtained by determining at 
each frequency the mean, 5th, and 95th percentiles of the 600 spectrum values. Also presented on Fig. 6 is the response of the mean model (dashed lines). Note in this figure that the 5th-95th percentile band increases with increasing frequency and is broader near the resonances and antiresonances of the mean model. Further, the response of the mean model is significantly lower than the 5th percentile near/at the antiresonances but very close to the 95 th percentile value in the neighborhood of the mean model resonant frequencies.

A similar analysis was next repeated for the nonlinear reduced order model subjected to the same excitation. The transverse displacement at the center of the beam obtained from the mean model is again shown both as function of time and frequency in Figs 7 and 8 for 12 inplane modes and 10 transverse. Note the significant reduction in the peak response (by approximately $40 \%$ ) as compared to the response of the linear system and that the peak displacement at the middle of the beam is of the order of twice the beam thickness. Important differences also occur in the frequency domain, more specifically broader and more numerous peaks as well as the disappearance of the sharp drop off at $2000 \mathrm{~Hz}$. A sample size of 600 realizations was again found well sufficient for convergence and led to the spectrum plot of Fig. 9. The observations drawn in connection with the linear system spectrum can be repeated here, i.e. the 5th-95th percentile band is largest at/near the peaks and valleys of the spectrum of the mean model thus forming complex shapes that may rapidly widen or narrow down. Since the location of the peaks of the response is a function of the response level of the system, it would be expected that the 5th-95th percentile band would shift to the right as the amplitude of the motions is increased. This expectation is confirmed on Fig. 10 which repeats the data of Fig. 9 as well as the 5th-95th band obtained with an excitation 2.25 times the one shown in Figs 1 and 2 which leads to a peak transverse displacement of approximately 3.5 thicknesses at the beam middle for the mean model (computations carried out with 12 transverse modes and 12 in-plane). 
Note as well the distortion of the uncertainty band as it shift to higher frequencies.

The coefficients $\bar{S}_{i j}, \hat{S}_{i j}^{(m)}$, and $\widetilde{S}_{i j}^{(m, n)}$ corresponding to the stress $\sigma_{x}$ at the middle of the beam were also obtained by the STEP algorithm and enabled the analysis of the effects of uncertainty on this stress. Shown in Fig. 11 is the comparison of the 5th-95th percentile bands at the two nonlinear response levels of Fig. 10. The discussion carried out in comparison with this figure holds here as well, the band is distorted as it moves to the right due to higher response level. A comparison of the uncertainty bands of the displacement and stress (Figs 10 and 11) indicate that the latter one is much more complex than the former one due to the large number of peaks of the spectrum, which itself results from the quadratic transformation of Eq. (28).

Uncertainty in the natural frequencies may originate from either the stiffnesses and/or the masses. In this light, it was desired to compare the uncertainty band associated with the $4 \%$ mean square variation of the natural frequencies assuming that it originates from stiffnesses alone (as done above) or masses alone. This comparison, shown in Fig. 12, demonstrate that the two 5th95th percentile bands are very close together with the one associated with mass uncertainty typically slightly broader than its stiffness counterpart. This observation may be justified by considering a uniform relative change of all stiffnesses by a value $\alpha$. Such a change may be reflected as a uniform change by $1 / \alpha$ of all stiffnesses, linear and nonlinear. With $\alpha$ an uncertain variable, this observation would suggest that the randomness in mass would be similar to a randomness in the stiffnesses in which there exists a strong positive correlation between the various linear and nonlinear terms, i.e. increases/decreases in the linear stiffness terms being matched by increases/decreases of the nonlinear terms. Such a positive correlation does imply a lower 5th percentile of the response and a higher 95th percentile, i.e. a broader band, as compared to the case in which linear and nonlinear stiffnesses do not exhibit a significant correlation as tends to be the case in the uncertain stiffness computations. Similar observations 
can be drawn from the uncertainty bands in the stress spectrum (not shown here for brevity).

The above discussions have all been relevant to a broad band excitation, i.e. similar to Figs 1 and 2 and it was wondered whether similar results would also be seen for a narrowband excitation, i.e. in the range $[1000,1500] \mathrm{Hz}$ see Figs 13 and 14. Given the previous observations, only the effects of uncertainty on the stiffness tensors $\underline{\underline{K}}^{(1)}, \underline{\underline{K}}^{(2)}$, and $\underline{\underline{K}}^{(3)}$ were considered. The computations were carried out with 16 transverse modes and 12 in plane modes at a time step of $\Delta t=2.510^{-5} \mathrm{~s}$ and the computations were carried out for $n_{t o t}=30000$ time steps. Shown in Figs 15 and 16 is the transverse displacement of the mid point of the beam in both time and frequency domains. Clearly, the excitation favors the fifth linear mode (symmetric mode of frequency equal to $1055 \mathrm{~Hz}$ ) and its response is significantly nonlinear leading to the broad response peak seen in Fig. 16. Note as well the presence of sharper peaks at smaller energy levels associated with the first and third modes which are out of band but are excited by the nonlinear interaction of the modes. The introduction of uncertainty in the linear and nonlinear stiffness tensors leads to the spectrum plot of Fig. 17. Surprisingly, it is found that the dominant peak is quite robust but that a very large uncertainty band occurs in conjunction with the first mode. In fact, the 95th percentile of the spectrum at this first peak is at the same energy level than that of the dominant peak. Thus, uncertainty on this narrowband response manifest itself mostly by a potentially dramatic increase in the component of response associated with the first linear mode.

\section{SUMMARY}

The focus of this investigation has been on the formulation of a general methodology for the consideration of both parameter and model uncertainty in the modeling of geometrically nonlinear dynamic systems. It was argued first that the most appropriate framework for the 
inclusion of model uncertainty is in terms of reduced order models of the system, especially those which are built from a deterministic basis, e.g. the linear modes of the mean model.

On this basis, a comprehensive analysis was undertaken to clarify the derivation of such reduced order models, first from the governing equations of a linearly elastic continuum. An important aspects of this effort was to highlight the fundamental properties of the various tensors involved in the reduced order model as these properties will have to be satisfied for every realization of the uncertain reduced order model. Symmetry properties of the mass and the three stiffness tensors were first stated. Next, the positive definiteness of the stiffness tensors $\underline{\underline{K}}^{(1)}$ and $\underline{\underline{K}}^{(3)}$ was recognized but this statement was shown to be resulting from a stronger positive definiteness property that involves all three stiffness tensors, i.e. of the matrix $\underline{\underline{K}}_{B}$ of Eq. (24). The derivation of a reduced order model of a geometrically nonlinear system from a computational model (e.g. finite element model) of it was also addressed and a recently devised approach (the STEP algorithm) was reviewed in details. It was finally noted that a complete reduced order model should also include the characterization of the stress field and this task was also achieved.

The stochastic modeling of uncertainty (parameter and model) within the context of the reduced order model was considered next. It was highlighted that such a modeling cannot be achieved in an ad-hoc manner but rather should be deep rooted in stochastic mechanics to achieve the generality and accuracy desired. To this end and following recent work in this area, a nonparametric approach was adopted in which the joint distribution of the coefficients of the reduced order model was not postulated but rather derived according to the maximum entropy principle under the constraints of symmetry and positive definiteness demonstrated earlier. The consideration of uncertainty in mass, damping, and stiffness according to this nonparametric 
approach was described in detail and was found to be computationally advantageous (owing to expedient algorithms for the simulation of samples of the reduced order model coefficients) and appealing in practical applications (because it requires only one measure of dispersion to characterize the uncertainty). Further, the nonparametric approach leads to reduced order models in which all coefficients are uncertain and are generally dependent on each other, as might be expected from an uncertain system.

A slender beam was finally considered to exemplify the methodology presented and its specific mean reduced order model was first derived by the STEP algorithm from a full finite element model. Next, uncertainty in mass and stiffness were considered one at a time to demonstrate the application of the nonparametric methodology and compare the effects of these different types of uncertainty on both displacements and stresses inside the beam. These results were found to be in good agreement with physical expectations.

\section{REFERENCES}

1. Ewins, D.J., "The Effects of Detuning upon the Forced Vibrations of Bladed Disks," Journal of Sound and Vibration, Vol. 9, pp. 65-79, 1969.

2. Ghanem, R., and Spanos, P.D., Stochastic Finite Elements: A Spectral Approach, SpringerVerlag, New York, 1991.

3. Soize, C., "A Nonparametric Model of Random Uncertainties on Reduced Matrix Model in Structural Dynamics," Probabilistic Engineering Mechanics, Vol. 15, No. 3, pp. 277-294, 2000.

4. Soize, C., "Maximum Entropy Approach for Modeling Random Uncertainties in Transient Elastodynamics," Journal of the Acoustical Society of America, Vol. 109, No. 5, pp. 1979-1996, 2001.

5. Soize, C., "Random Matrix Theory for Modeling Uncertainties in Computational Mechanics," Computer Methods in Applied Mechanics and Engineering, Vol. 194, pp. 1333-1366, 2005.

6. Soize, C., "Probabilistic Models for Computational Stochastic Mechanics and Applications," Proceedings of the 9th International Conference on Structural Safety and Reliability ICOSSAR'05, Rome, Italy, 19-23 June 2005, Augusti, G., Schueller, G.I., and Ciampoli, M., (Eds). Millpress, Rotterdam, Netherlands, pp.23-42.

7. Muravyov, A.A., and Rizzi, S.A., "Determination of Nonlinear Stiffness with Application to Random Vibration of Geometrically Nonlinear Structures," Computers and Structures, Vol. 81, No. 15, pp. 1513-1523, 2003.

8. Mignolet, M.P., Radu, A.G., and Gao, X., "Validation of Reduced Order Modeling for the Prediction of the Response and Fatigue Life of Panels Subjected to Thermo-Acoustic Effects", Proceedings of the 8th International Conference on Recent Advances in Structural Dynamics, Southampton, United Kingdom, Jul. 14-16, 2003. 
9. Radu, A., G., Yang, B., Kim, K., and Mignolet, M.P., "Prediction of the Dynamic Response and Fatigue Life of Panels Subjected to Thermo-Acoustic Loading," Proceedings of the 45th Structures, Structural Dynamics, and Materials Conference, Palm Springs, California, Apr. 1922, 2004.

10. Hollkamp, J.J., Gordon, R.W., and Spottswood, S.M., "Nonlinear Modal Models for Sonic Fatigue Response Prediction: A Comparison of Methods," Journal of Sound and Vibration, Vol. 284, No. 3-5, pp. 1145-1163, 2005.

11. Fung, Y.C., and Tong, P., Classical and Computational Solid Mechanics, World Scientific, River Edge, New Jersey, 2001.

12. Bonet, J., and Wood, R.D., Nonlinear Continuum Mechanics for Finite Element Analysis, Cambridge University Press, Cambridge, 1997.

13. Devroye, L., Non-Uniform Random Variate Generation, Springer Verlag, New York, 1986.

14. Mignolet, M.P., and Soize, C., "Nonparametric Stochastic Modeling Of Linear Systems With Prescribed Variance Of Several Natural Frequencies," Probabilistic Engineering Mechanics, In Press.

15. Soize, C., "Construction of Probability Distributions in High Dimension using the Maximum Entropy Principle. Applications to Stochastic Processes, Random Fields and Random Matrices," International Journal for Numerical Methods in Engineering, Submitted.

16. Bathe, K.-J., and Wilson, E.L., Numerical Methods in Finite Element Analysis, Prentice-Hall, Englewood Cliffs, New Jersey, 1976. 